\title{
The Effect of Malaria Integrated Supportive Supervision (Miss) on Knowledge and Practice of Malaria Prevention and Management: A Comparative Study of Miss Supported and Non Supported Primary Health Facilities in Nasarawa State, Nigeria
}

\author{
Article by Alabi Babatunde \\ Public Health, Texila American University, Nigeria \\ E-mail: tunnybabsmed@yahoo.com
}

\begin{abstract}
Background: Malaria is a major cause of morbidity and mortality in Nigeria, directly contributing to poverty, low productivity, and reduced school attendance. The mosquito-borne illness causes over 100 million clinical cases every year. It accounts for about $60 \%$ of all outpatient attendances and $30 \%$ of all hospital admissions and is responsible for the annual death of 300,000 children under 5 years as well as $11 \%$ of maternal mortality cases reported each year. In children, malaria is responsible for $25 \%$ of all infant-related mortality and 30\% of child-related mortality. (National Malaria Control Strategic Plan FMOH 2009-2013)

Integrated Supportive Supervision (ISS) is a harmonized supervisory system which uses a common tool and reporting format based on a collection of indicators from as many work processes/initiatives/programmes as possible. It is driven by a common supervisory team usually working as several sub-teams, thus ensuring that managers are in the field on a regular basis (monthly or quarterly) to check the performance of subordinates and help them to improve on their competencies and output. What is also key besides getting managers into the field on a regular basis and thus understanding the context and challenges within which health workers function, is the importance of both supervisors and supervisees agreeing on a plan of action to improve health care delivery. This plan of action must include activities/actions by both the supervisor and the supervisee. This is critically important and can be used to measure progress on subsequent visits.

This study aimed at evaluating the effect Malaria Integrated Supportive Supervision (MISS) on Malaria Service Delivery in MISS supported Primary Health Facilities and Non-Supported Primary Health Facilities across various LGAs in Nasarawa State.

Materials and Methods: This was a facility-based interventional study with MISS intervention and MISS non- intervention groups. The study subjects were Officers in charge of each Primary Health Facility, selected through a multistage sampling technique and data collection was done using semi structured self-administered questionnaire, all data were entered in an Excel sheet and analyzed using SPSS version 17.0. Paired t test was used in testing for significance between intervention and nonintervention group.

Results: On the Knowledge and Practice of malaria prevention and vector control, the mean score for the MISS and Non MISS groups are; 92.98 and 53.41respectively. The two-tailed P value equals 0.0395 which by conventional criteria, this difference is considered to be statistically significant.

Mean score on Diagnosis and Treatment knowledge and practice between the MISS intervention and Non-MISS intervention groups are; 81.49 and 66.53 respectively. The two-tailed $P$ value equals 0.0642 . By conventional criteria, this difference is considered not to be quite statistically significant. This could however be attributed partly to the fact that trainings on diagnosis and treatment was held jointly among the two groups.
\end{abstract}


DOI: $10.21522 /$ TIJPH.2013.05.04.Art008

ISSN: $2520-3134$

Malaria in Pregnancy mean score between the MISS intervention and Non-MISS Intervention groups was 86.38 and 66.10 respectively. The two-tailed $P$ value equals 0.0138 which by conventional criteria, this difference is considered to be statistically significant

Conclusion: This study has demonstrated that integrated supportive supervision is an effective tool of improving knowledge and practice of malaria prevention and case management in Primary Health Facilities

\section{Introduction}

Malaria is an infectious disease caused by a parasite, Plasmodium, which infects red blood cells. Malaria is characterized by cycles of chills, fever, pain, and sweating. Historical records suggest malaria has infected humans since the beginning of mankind (Snow et.al 1999). The name "mal aria" (meaning "bad air" in Italian) was first used in English in 1740 by H. Walpole when describing the disease. The term was shortened to "malaria" in the 20th century. Laveran (1880), was the first to identify the parasites in human blood while in 1889 Ross discovered that mosquitoes transmits malaria. Of the four common species that cause malaria, the most serious type is Plasmodium falciparum malaria. It can be lifethreatening. However, another relatively new species, Plasmodium knowlesi, is also a dangerous species that is typically found only in long-tailed and pigtail macaque monkeys. Like $P$. falciparum, $P$. knowlesi may be deadly to anyone infected. The other three common species of malaria ( $P$. vivax, $P$. malariae, and $P$. ovale) are generally less serious and are usually not life-threatening. It is possible to be infected with more than one species of Plasmodium at the same time (Ross 1989).

Currently, about 2 million deaths per year worldwide are due to Plasmodium infections. The majority occur in children under 5 years of age in sub-Saharan African countries. There are about 400 million new cases per year worldwide (Craige 1999).

Malaria is a major cause of morbidity and mortality in Nigeria too, directly contributing to poverty, low productivity, and reduced school attendance. The mosquito-borne illness causes over 100 million clinical cases every year. It accounts for about $60 \%$ of all outpatient attendances and $30 \%$ of all hospital admissions and is responsible for the annual death of 300,000 children under 5 years as well as $11 \%$ of maternal mortality cases reported each year, (National Malaria Control Strategic Plan FMOH 2009-2013). In children, malaria is responsible for $25 \%$ of all infant-related mortality and $30 \%$ of child-related mortality.

The Government of Nigeria has adopted a proven malaria control strategy that includes protection against mosquito bites, prompt and correct diagnosis and treatment of malaria cases, and the provision of intermittent preventive therapy (IPT) to pregnant women amongst others. At the same time, there is a concerted effort to strengthen the capacity and management systems by the National Malaria Control Programme (NMCP). However, many structural and behavioral barriers prevent the effective implementation of this national strategy.

The USAID-funded 'Malaria Action Program for States' (MAPS) has since 2012 supported the Nasarawa State Malaria Control Programme (SMCP) establish and strengthen the state's health sector supervisory system at both the LGA and state levels. Subsequently, the State has been supported to plan and conduct quarterly Malaria-focused Integrated Supportive Supervision exercises in 7 LGAS out of the 13 LGAS in Nasarawa State. These quarterly MISS exercises have in many ways informed malaria control approaches and intervention strategies in the state. In addition, some of the key management and operational findings from the healthcare facilities and LGA PHC department's engagement processes for malaria control are documented and reported for possible policy responses and higher management level attention and actions. Also the on-the-job-capacity building (OJCB) and mentoring aspects of supportive supervision exercises have continuously increased healthcare providers' knowledge and skills. 
In Nasarawa State, malaria is one of the most common reasons for hospital visits in all age groups. It is also estimated that half of the population of the State experience at least one episode of malaria attack in a year, while under-5 children have an average of 2-4 episodes annually, (World Malaria Report' 2012).

Various interventions have been proposed to tackle health worker poor performance. Prominent among them is the strengthening of supervision which has been recognized as a generally effective intervention in improving health worker performance in low resource settings, (Rowe et., al, 2005). Supervision has been reported as one of the nonmonetary motivators of health workers and its absence can be correlated with health worker poor job satisfaction as well as poor service delivery, (Chirdan, et, el 2008).

Efforts to strengthen health systems often have little effect, because support, follow-up and supervision which are critical to the implementation of strategies are not done properly. In many places where some supervision is done at all, they are undertaken by one officer who makes a brief and unscheduled check on subordinates with little or no feedback. Apart from issues around the skills and process of supervision, many programmes and interventions in the health sector are designed and implemented with separate supervisory arrangements. The result is the existence of parallel supervisory systems which create the possibility of duplications and wastage and the inability to maximize potential synergies between the various initiatives.

Integrated Supportive Supervision (ISS) is a harmonized supervisory system which uses a common tool and reporting format based on a collection of indicators from as many work processes/initiatives/programmes as possible. It is driven by a common supervisory team usually working as several sub-teams, thus ensuring that managers are in the field on a regular basis (monthly or quarterly) to check the performance of subordinates and help them to improve on their competencies and output. What is also key besides getting managers into the field on a regular basis and thus understanding the context and challenges within which health workers function, is the importance of both supervisors and supervisees agreeing on a plan of action to improve health care delivery. This plan of action must include activities/actions by both the supervisor and the supervisee. This is critically important and can be used to measure progress on subsequent visits.

In this study, Malaria integrated supportive supervision in the context of knowledge and practice of prevention and case management of malaria was studied in Primary Health Facilities. Since Malaria is responsible for majority of outpatient consultations in Nigeria (FMOH; 2010). Health Workers in various capacities in PHCs see more cases of malaria than any other disease; hence malaria service delivery provides an appropriate platform to measure the effect of supportive supervision of Health workers.

\section{Research methodology}

This chapter discusses the research design, study settings, target population, sampling technique, instrument for data collection, validity of instruments, method of data collection and analysis of the data constrains and ethical consideration.

\section{Research design}

This study employed comparative design. This design involves the use of self-administered questioners to the OICs of selected primary health facilities across both MISS supported and non-supported LGAs in Nasarawa State and thereafter, comparing the knowledge and practice of malaria prevention and case management of malaria.

\section{Study settings}

There are three tiers of health care services in Nasarawa State, namely primary, secondary and tertiary. The State Ministry of Health $(\mathrm{SMoH})$ initiates policies and supervises the overall health system in the state. The State Hospitals Management Board (HMB) coordinates and supervises the day to day management of all public secondary health care facilities. The State Primary Health Care Development 
DOI: $10.21522 /$ TIJPH.2013.05.04.Art008

ISSN: $2520-3134$

Agency (SPHCDA) was established in 2009 to supervise and coordinate the activities at the Primary Health Care (PHC) level. Two tertiary health institutions also exist in the state namely the Dalhatu Araf Specialist Hospital (DASH), Lafia and the Federal Medical Centre, Keffi.

The Malaria integrated supportive supervision (MISS) system is functional only in 168 Malaria Donor Funded supported facilities in seven (7) LGAs out of the 13 LGAs. The State has a total of 1100 Public health facilities (788 PHCs, 18 SHC facilities, 2 tertiary health facilities) and 292 private health facilities. (Nasarawa State Health Strategic Plan, 2009).

Nasarawa State is one of the 36 States in the Federal Republic of Nigeria. It came into existence on the first of October, 1996. The State lies between latitude $7^{\circ} 45^{\prime}$ and $9^{\circ} 25^{\prime}$ North of the Equator and between longitude $7^{\circ}$ and $9^{\circ} 37^{\prime}$ East of the Greenwich Meridian, (FMoH, 2009). It is situated in the North-Central Geo-political Zone of Nigeria, otherwise known as the Middle Belt. It has a land area of 27,116.8 square kilometers with a population of 1,863,275 according to 2006 provisional census, and the 2015 projected population of 2,473,969 (National Population Commission, 2006).

It has 13 Local Government Areas and 147 constituent wards. The State is bounded by Kaduna State in the North, Plateau in the North East, Taraba State in the South East, Benue State in the South, Kogi State in the South West and Federal Capital Territory in the West. Apart from these States as boundaries, river Benue forms part of its South West. The State has many ethnic groups. While it is difficult to draw out a neat ethnic map of the state, several villages are predominantly homogenous ethnic groups. The major ethnic groups include Eggon, Mada, Alago, Rindre, Gwandara, Migili (Koro), Gbagyi, Egbura, Agatu, Bassa, Afo, Kantana, Akye, Kanuri, Tiv, Hausa, Fulani and Nyankpa (Yeskwa), ( Nasarawa State Health Strategic Plan, 2009).

The majority of the inhabitants of the State engage in both crop and animal farming. Some are petty traders with a small proportion being civil servants. The State owns tertiary institutions such as the Nasarawa State University and the School of Health Technology both located in Keffi, College of Education Akwanga, Nasarawa State Polytechnic, College of Agriculture and the School of Nursing, all located in Lafia. The Federal University, National Open University of Nigeria and the Federal Polytechnic also located in Lafia and Nasarawa Eggon towns respectively. There is also the Bingham University (a private university) which is located at Karu. There are many tourist sites such as the Keana salt pond, Doma dam and the Farin Ruwa waterfalls. The State is also blessed with many solid minerals in large quantities which earned it the appellate "Home of Solid Minerals".

\section{Target populations}

The population under study consists of OICs of PHCs facilities in the thirteen (13) Local Government Areas of Nasarawa State.

\section{Sample size, sampling technique and method of data collection}

Nasarawa State has 13 Local Government Areas and 147 constituent wards. The State has 13 LGA and a total of 1100 Public health facilities (788 PHCs, 18 SHC facilities, 2 tertiary health facilities).

Donor projects in the state currently supports a total of 23 PHC and 1SHF in each of the 13 LGAs, making a total of 312 facilities in malaria prevention, diagnosis, treatment, monitoring and evaluation, advocacy communication and social mobilization and health information management systems.

The Donor project has been supporting the implementation of MISS in 7 LGAs out of the 13 LGAs which gives a total of 168 MISS supported facilities out of the 312 facilities being supported.

\section{Stage one: selection of study groups (MISS Intervention and Non MISS Intervention)}

Stratified random sampling is a method of sampling where the population is divided into two or more groups which are known to have the same features relating to the phenomenon being studied (Grady, et al. 2008). 
The method adopted (stratified sampling method) was to reduce problems associated with data collection. Qualitative sampling methods, which due to the peculiar nature of the study site and subjects, involved strategies that combined different (mixed purposeful) sampling techniques were applied in this study. For this work non-probability sampling methods, in particular, convenience sampling using the mixed purposeful approach with maximum variation (best case, worst case) as described by( Johnson and Christensen, 2003) was used. Using stratified sampling technique, we first divide the LGAs into MISS intervention (Group A-7 LGAs) and Non MISS intervention (Group B-6LGAs). This will enable us to draw inferences about each group that may be lost in a more generalized random sample. In this study we are looking at two strata in a population: PHCs where MISS is been conducted and PHCs where it is not being conducted.

\section{Stage two: selection of LGAs}

2 LGAs will be selected randomly from each of the Strata (MISS supported and none supported), giving a total of 4 LGAs study population shared equally between both strata.

\section{Stage three: selection of facilities and administration of questionnaire/tool}

All the Supported PHCs in each LGA; (23 PHCs each) will be sampled, giving a total of 92 Health Facilities out of the 312 facilities with equal donor support (excluding MISS).

\section{Stage four: questionnaire administration}

A self-administer semi structured questionnaire will be administered to the Officer in-charge (OIC) of each facility, accessing the various malaria service delivery thematic areas.

\section{Primary sources}

The primary sources of data include the researcher and questionnaire administered

\section{Secondary sources}

The secondary sources of data are those that were obtained from published and unpublished papers which were utilized by the researcher for the literature review and theoretical framework of the project. They also provide alternative additional data on issues relevant to the study and some, of which the researcher may not be able to personally source.

The study employed the use of structured questionnaires to obtain data from the population sample in support of the study. The questionnaire was designed according to Likert3 pattern. Respondents were required to choose from any of the responses.

Validity can be defined as the degree to which a test measures what it is supposed to measure. There are three basic approaches to the validity of tests and measures as shown by Mason and Bramble (1989). These are content validity, construct validity, and criterion-related validity. This Study adopted.

\section{Method of data analysis}

All data were entered in an Excel sheet and analyzed using SPSS version 17.0. Paired t test was used in testing for significance between MISS intervention and non-MISS intervention.

\section{Results}

92 Officers in-charge of selected PHCs across 4 LGAs are respondent to the questions. The results are reflections of the respondent's views to questions in the tool. These results are presented as tables, analyzed and discussed below.

In this study, the following scoring systems were modified and adopted. In calculating knowledge scores, a point was awarded for each correct response and no point for wrong responses and non- 
DOI: $10.21522 /$ TIJPH.2013.05.04.Art008

ISSN: $2520-3134$

responses. The percentage of respondent who got each question correctly was calculated for each of the study group, and the average cumulative percentage right scores for each of the study thematic areas.

\section{Knowledge and practise of malaria prevention and vector control}

92 respondent divided into 46 MISS supported PHCs and 46 none supported PHCs were sampled on their knowledge and practice of malaria prevention and vector control. As shown in Table 4.1 below, the average correct score for the 46 MISS supported facilities on Knowledge and Practice of malaria prevention and vector control is $91.38 \%$ and for MISS Non-supported PHCs is $59.08 \%$

The result of respondent on the channels of distribution of LLINs in their facility was strikingly significant, as the MISS supported facilities engage all the channels as prescribed in the National Malaria Strategic Plan (NMSP and had a 100\% score while the MISS Non supported only engage some of the recommended channels and not all and only $2.2 \%$ of respondent were doing the recommended. The level of significance was set at 0.05 . The two-tailed $\mathrm{P}$ value equals 0.0395 ; this difference is considered to be statistically significant

\section{Knowledge and practise of malaria diagnosis and treatment}

92 respondents divided into 46 MISS supported PHCs and 46 Non-supported PHCs were sampled on their knowledge and practice of malaria diagnosis and treatment. As shown in Table 4.2 below, the average correct score for the 46 MISS supported facilities on Knowledge and Practice of malaria diagnosis and treatment is $81.49 \%$ and for MISS Non-supported PHCs is $66.53 \%$. The two-tailed P value equals 0.0642 ; this difference is considered to be not quite statistically significant.

\section{Knowledge, practise and management of malaria in pregnancy}

92 respondents divided into 46 MISS supported PHCs and 46 Non-supported PHCs were sampled on their knowledge, practice and management of malaria in pregnancy. As shown in Table 4.3 below, the average correct score for the 46 MISS supported facilities on Knowledge, Practice and Management of malaria in pregnancy is $86.38 \%$ and for MISS Non-supported PHCs is $66.1 \%$. The two-tailed P value equals 0.0138 ; this difference is considered to be statistically significant.

\section{Discussion}

This study so far investigated the effect of malaria integrated supportive supervision (MISS) on knowledge and practice of malaria prevention and management between MISS supported and Non-MISS supported PHCs across various LGAs in Nasarawa State.

The specific objectives were; to compare the knowledge and practice of Malaria Prevention and Vector control among the intervention and control groups, to compare the knowledge and practice of care providers on Diagnosis and Treatment of Malaria among the two groups and to access the knowledge and practice of the study groups on management of malaria in Pregnancy.

The study found out that the implementation of Malaria Integrated Supportive Supervision (MISS) has significant effect on the knowledge and practice of malaria prevention and vector control of the supported PHCs, worthy of note is the fact that all MISS supported facilities engage all the NMSP routine distribution channels for the distribution of LLINs, their knowledge of the benefit, care and proper use of the LLIN was also significant as compared to the MISS Non-supported PHCs.

On the knowledge and practice of malaria diagnosis and management, both groups have comparably close scores. This could be attributed to the fact that trainings conducted on diagnosis and treatments of malaria are not separated between MISS supported and none supported LGAs.

Knowledge and practice of management of malaria in pregnancy was significantly higher in the intervention group compared to the control group. All pregnant women in the MISS supported PHCs were given SP, a large proportion were also given as DOT and the recommended number of doses according to 
the National guideline. Also the line of management according to the National Malaria Guideline of positive malaria cases in pregnancy was significantly higher in the intervention group. These findings in knowledge improvement on malaria service delivery can cascade into an overall improvement in the complement of services rendered by Healthcare worker. Notable among these is the generation of quality service data by health workers.

Effective SS also facilitates generation of quality health service data and improves the use of such data to inform action. This was evident in Kano State, Nigeria, where 91\% of HF that had SS visits demonstrated an improved data consistency across data tools. Also, 92\% of HFs in which one person attended the most recent LGA review meeting had improved data consistency between tally sheet and immunization monthly summary sheets (Kano DQUSS report, 2015).

Furthermore, SS has been shown to improve data accuracy through a demonstrated study conducted in South Africa (Mphatswe, 2011). Main finding revealed that data accuracy improved from 37\% to 65\% after intensive SS. Marshall and Fehringer (2013) conducted a series of interviews and direct observations related to SS in Haiti. Most participants attributed the successes achieved in data quality to standardized data collection tools, ongoing supervision, and on-the-job training.

\section{Conclusions}

One of the important cornerstones of Supportive Supervision (SS) is working with health staff to establish goals, monitor performance, identify and correct problems, and proactively improve the quality of service and service data generated. Together, the supervisor and health workers identify and address weaknesses on the spot, thus preventing poor practices from becoming routine. Supervisory visits are also an opportunity to recognize good practices and help health workers to maintain their high-level of performance. According to Marquez and Kean (2002), SS was described as a process that promotes quality at all levels of the health system by strengthening relationships within a system, focusing on the identification and resolution of problems, and helping to optimize the allocation of resources by promoting high standards, teamwork, and better two-way communication while also helping staff to improve their own work performance continuously. It is carried out in a respectful and non-authoritarian way with a focus on using supervisory visits as an opportunity to improve knowledge and skills of health staff. It focuses on monitoring performance towards goals, and using data for decision-making, and depends upon regular follow-up with staff to ensure that new tasks are being implemented correctly.

This study has demonstrated that supportive supervision is a feasible intervention in increasing malaria prevention/vector control, diagnosis and treatment and prevention and management of malaria in pregnancy in PHCs workers as well as increase healthcare performance. It also showed that, given sufficient management support, malaria prevention and case management practices have the potential to improve tremendously following supportive supervision.

\section{Recommendation}

Supportive supervision should therefore be incorporated into the existing frameworks for improving healthcare worker performance in the Country. This can be achieved if Health Ministries ensure that a yearly budget line is created for this activity in the Annual Operational Plan for health programs. This will also ensure regular on the job mentoring and capacity building for healthcare workers towards continuous quality improvement in health care delivery. There is also an urgent need for further studies on supportive supervision of malaria prevention and case management that may lead to an improvement in the strategy for supportive supervision. 
DOI: $10.21522 /$ TIJPH.2013.05.04.Art008

ISSN: $2520-3134$

\section{References}

[1].Alphonse Laveran (1880) in Nye Edwin (2002) Discoverer of the malaria parasite and Nobel Laureate. Journal of medical biology.

[2].Bradley, J. et al., (2002) COPE® for Child Health in Kenya and Guinea: An Analysis of Service Quality, Engender Health, New York.

[3].Cronbach, 1. J. et el (1955) The counselor's problems from the perspective of

[4].Communication theory, Minnesota Press

[5].Chirdan Oo, Zoakah Ai, Ejembi Cl. (2008), Impact of health education on home treatment and prevention of malaria in Jengre, North Central Nigeria. Ann Afr Med

[6].Craige Mh, Snow Rw, \& Sueur D (1999) A climate based distribution model of malaria transmission in subSaharan Africa. Parasitilogy today.

[7].Dickson, j. A. (1977) the Effects of Hyperthermia in Animal Tumor Systems. In Selective Heat Sensitivity of Cancer Cell.

[8].Deobold b. Van dalen mcgraw-hill, (1979) Understanding Educational Research: an introduction

[9].Dohlie, mb. Et al., (2002), Empowering Frontline Staff to Improve the Quality of Family Planning Services: A

Case Study in Tanzania., Responding to Cairo, Case Studies of Changing Practice in Reproductive Health and Family Planning, Population Council, New York.

[10]. Dohlie, M., (2001) Supportive Supervision, Facilitative Supervision Handbook, New York, 2001.

[11]. Federal ministry of health. National policy on malaria diagnosis and treatment. Fmoh; (2010).

[12]. Framework for the coordination of malaria control program in Nigeria, fmoh, (2009).

[13]. Gavi implementation task force, monitoring national immunization systems using core indicators, (12002).

Government of India, Child Health Division, Department of Family Welfare, Ministry of Health \& Family Welfare,

Introduction of Hepatitis B Vaccine in the Universal Immunization Programme: A Handbook for Programme Managers \& Medical Officers.

[14]. Gemperli a, vounatson p, sogoba $\mathrm{n}$ et. al (2006), malaria mapping using transmission model. American journal of epidemiology.

[15]. Grady, c. et al. (2008) "Research Benefits for Hypothetical HIV Vaccine Trials: The Views of Ugandans in the Rakai District." IRB: Ethics and Human Research

[16]. Kim, y.m. et al., (2000) The Quality of Supervisor-provider Interactions in Zimbabwe, Operations Research Results, USAID Quality Assurance Project, Bethesda, Maryland.

[17]. Kiran, v., (2002) Supervision and Monitoring in AP Immunization Strengthening Program, draft.

[18]. Management Sciences for Health, Improving Supervision: A Team Approach, the Manager. Electronic Resource Center. Available online at www.erc.msh.org.

[19]. Management sciences for health, Lessons from the Field: Performance Improvement Case Study. Ashonplafa. Available online at www.pihealthcare.org.

[20]. Mason, ej \& bramble, wj (1989) "Understanding and Conducting Research Applications in Education and Behavioural Sciences”. New Delhi: McGraw Hill Book Co.

[21]. Management sciences for health, Supervising and Supporting Your Staff, The Manager. Electronic Resource Center. Available online at www.erc.msh.org.

[22]. Marquez, L. and L. Kean, (2002) Making Supervision Supportive and Sustainable: New Approaches to Old Problems, Maximizing Access and Quality Initiative, MAQ Paper No. 4, USAID, Washington, DC.

[23]. Mcmahon, r. et al., (1992) On Being in Charge: A Guide to Management in Primary Health Care, World Health Organization, Geneva, 1992.

[24]. Mutabaruka, e. et al., (2002) Integrated Training and Supportive Supervision: A Challenge to Improved Quality of Health Services in Countries.

[25]. Mutombo m, (2002) Supervision du PEV (Présentations). 
Texila International Journal of Public Health

Volume 5, Issue 4, Dec 2017

[26]. Managers, preliminary version, who African regional office, March (2003). Who, Supervision: An Underutilized Management Tool to Identify Risk Areas, EPI Newsletter Expanded Program on Immunization in the Americas, Volume XXIV.

[27]. National population commission, (2006).

[28]. Nasarawa state health strategic plan, (2009).

[29]. National malaria control strategic plan fmoh (2014-2020)

[30]. World malaria report' (2012).

[31]. National immunisation program, national mch centre, ministry of health, (2003) Cambodia, Monitoring and Management Support Strategy, Immunisation Coverage Improvement, Cambodia.

[32]. Plackett, r. 1. (1983). "Karl Pearson and the Chi-Squared Test". International Statistical Review (International Statistical Institute

[33]. Prime ii, stages, steps and tools (2003): A Practical Guide to Facilitate Improved Performance of Healthcare Providers Worldwide.

[34]. Prime ii, transfer of learning (2003): A Guide to Strengthening the Performance of Health Care Workers.

[35]. Rowe AK, de savigny d, lanata cf, victora cg. (2005) how can we achieve and maintain high-quality performance of health workers in low-resource settings? Lancet.

[36]. Ronald Ross (1989) malaria the mosquito connection. Vigyan prasar science portal.

[37]. Salem, b. et al., (1996) Facilitative Supervision: A Vital Link in Quality Reproductive Health Service Delivery, AVSC Working Paper.

[38]. Snow rw, craige mh, deichman u. et.al (1996) A Continental risk Map for malaria mortality among African children. Parasitology today.

[39]. Tukey, j. w. (1977). EDA. Reading, Mass.: Addison-Wesley, p. 43

[40]. World Health Organization (2003), Immunization in Practice (unpublished draft 4).

[41]. WHO, Module 21 (2003): Supervision for EPI Managers, Mid-Level Management Course for EPI

[42]. WHO, (2002) Supportive Supervision (Presentation), Meeting of the GAVI Implementation Task Force on Supportive Supervision, Geneva.

[43]. Willmott, a.s. and nuttall, d.1. (1975) the Reliability of Examinations at London: Macmillan Educat 2.9

[44]. Marquez 1, Kean 1. (2002). Making Supervision Supportive and Sustainable: New Approaches to Old Problems, Maximizing Access and Quality Initiative [MAQ Paper No. 4]. Washington, DC: U.S. Agency for International Development.

[45]. Mphatswe. (2011). improving public health information: a data quality intervention in KwaZulu-Natal, South Africa. www.who.int/bulletin/volumes/90/3/11-092759.pdf.

[46]. Marshall a, fehringer j (2013). Supportive Supervision in Monitoring and Evaluation with Community-based Health Staff in HIV Programs: A Case Study from Haiti. Kano dqss report (2015). 